\title{
A Support Kernel Machine for Supervised Selective Combining of Diverse Pattern-Recognition Modalities
}

\author{
Alexander Tatarchuk ${ }^{1}$, Eugene Urlov ${ }^{2}$, Vadim Mottl $^{1}$, David Windridge ${ }^{3}$ \\ ${ }^{1}$ Computing Center of the Russian Academy of Sciences,Moscow, Russia \\ 2 Moscow Institute of Physics and Technology, Moscow, Russia \\ ${ }^{3}$ Centre for Vision, Speech and Signal Processing, \\ University of Surrey, Guildford, UK
}

\begin{abstract}
The Support Kernel Machine (SKM) and the Relevance Kernel Machine (RKM) are two principles of selective combining several object-representation modalities of any different kinds by means of incorporating the idea of supervised selectivity into the classical kernel-based SVM. The former principle consists in rigidly selecting a subset of presumably informative support kernels and excluding the others, whereas the latter one assigns positive weights to all of them. The RKM algorithm was completely elaborated in previous publications, whereas the known algorithm of implementing the SKM principle of selectivity supervision is applicable only to real-valued features. The present paper fills in this gap by harnessing the framework of subdifferential calculus for computationally solving the problem of constrained nondifferentiable convex optimization that occurs in the SKM training criterion applicable to arbitrary kernel-based modalities of object representation.
\end{abstract}

\section{Introduction}

In pattern recognition, the term "modality" is employed when speaking about a specific kind of mathematical computer-perceptible object representation. In terms of the measured modality, the hypothetical set of "all" real-world objects of interest $\omega \in \Omega$ is represented by the outputs of the respective sensor as generalized features $x(\omega) \in \mathbb{X}$ in some sensor-specific scale $\mathbb{X}$. In the simplest case, when the scale is the set of real numbers $\mathbb{X}=\mathbb{R}$, the objects are represented by values of a real numerical feature. Multimodal pattern recognition systems utilize several distinct feature modalities, often with different scales $\left(x_{i}(\omega) \in \mathbb{X}_{i}, i \in I=\{1, \ldots, n\}\right)$, to represent specific phenomena [1].

Feature scales $\mathbb{X}_{i}$ may be quite complicated, so that frequently the only way of treating real-world objects $\omega \in \Omega$ is via pair-wise comparison of their features $\left(x_{i}\left(\omega^{\prime}\right), x_{i}\left(\omega^{\prime \prime}\right)\right)$ using modality-specific functions $K_{i}\left(x_{i}^{\prime}, x_{i}^{\prime \prime}\right)$ defined in the respective scales $\mathbb{X}_{i} \times \mathbb{X}_{i} \rightarrow \mathbb{R}$. A function $K\left(x^{\prime}, x^{\prime \prime}\right)$ is said to be a kernel if it forms a semidefinite matrix for any finite collection of objects. It is well known that a kernel embeds the scale of the respective feature $\mathbb{X}_{i}$ into a hypothetical linear space $\tilde{\mathbb{X}}_{i} \supseteq \mathbb{X}_{i}$ in which it plays the role of inner product. 
In particular, when $x_{i}(\omega) \in \mathbb{X}_{i}=\mathbb{R}$, the natural kernel will be the product $K_{i}\left(x_{i}^{\prime}, x_{i}^{\prime \prime}\right)=x_{i}^{\prime} x_{i}^{\prime \prime}$. Support Vector Machines (SVMs), originally designed for two-class pattern recognition learning in $\mathbb{R}^{n}$, actually combine real-valued modalities by employing a joint kernel $K\left(\boldsymbol{x}^{\prime}, \boldsymbol{x}^{\prime \prime}\right)=\sum_{i=1}^{n} x_{i}^{\prime} x_{i}^{\prime \prime}$. This analogy is exploited by multi-kernel SVMs when more sophisticated kernel-represented modalities are to be combined [3-5].

When fusing several modalities of object representation, the necessity to moderate the inevitable overfitting threat makes it absolutely necessary to combine modality-specific features in a selective mode. We consider here the general case of kernel-induced feature scales $\left\{\tilde{\mathbb{X}}_{1}, \ldots, \tilde{\mathbb{X}}_{n}\right\}$ treated as hypothetical linear closures $\tilde{\mathbb{X}}_{i} \supseteq \mathbb{X}_{i}$ of arbitrary scales $\left\{\mathbb{X}_{1}, \ldots, \mathbb{X}_{n}\right\}$ with respective kernels defined over each of them $\left\{K_{i}\left(x_{i}^{\prime}, x_{i}^{\prime \prime}\right), x_{i}^{\prime}, x_{i}^{\prime \prime} \in \mathbb{X}_{i}\right\}$. The kernel-based approach removes the mathematical distinction between different kinds of feature scales $\tilde{\mathbb{X}}_{i}$, so that the kernel selection will boil down to the usual feature selection in the particular case of natively real-valued features $\tilde{\mathbb{X}}_{i}=\mathbb{X}_{i}=\mathbb{R}$.

There exist many feature (kernel) selection techniques classed in the literature as filters, which are applied to the feature set independently of classification technique, and wrappers, which consider feature selection in conjunction with classification [2].

It is the latter way of combining multiple kernels we keep to in this paper. More specifically, we further elaborate the methodology of selectivity supervision by a priori assigning the desired level of selectivity, ranging from the complete absence of selection to the adoption of only singular features. In our previous papers $[6,7]$, a way of achieving this range of behaviours was roughly outlined as the idea of incorporating selectivity into the two-class kernel-based Support Vector Machine.

Two principles of incorporating selectivity into the SVM proposed in [6] were called Support Kernel Machine (SKM) and Relevance Kernel Machine (RKM). The former principle consists in rigidly selecting a subset of presumably informative support kernels and excluding the others, whereas the latter one assigns positive weights to all of them.

An algorithm of implementing the RKM principle of selectivity supervision is completely elaborated in [6] and tested in [7] on the practical problem of signature verification by kernel-based fusing on-line and off-line modalities of signature representation. But as to the SKM principle, the algorithm described in [6] is applicable only to real-valued features $x_{i} \in \mathbb{X}_{i}=\mathbb{R}$.

The purpose of the present paper is to fill in this gap. The idea consists in harnessing the framework of subdifferential calculus [10] for computationally solving the problem of constrained nondifferentiable convex optimization that occurs in the SKM training criterion applicable to arbitrary kernel-based modalities of object representation. This approach allows to explicitly show the mechanism of selecting the support kernels and excluding the redundant ones relative to the given training set. 


\section{The Support Kernel and the Relevance Kernel Machines}

Let $\left\{\boldsymbol{x}_{j}=\left(x_{1 j}, \ldots, x_{n j}\right), y_{j}, j=1, \ldots, N\right\}$ be the training set of real-world objects $\left\{\omega_{j} \in \Omega, j=1, \ldots, N\right\}$ each of which is represented by the class-membership index $y_{j}=y\left(\omega_{j}\right) \in\{-1,1\}$ and the values of $n$ modality-specific features measured in the respective scales $x_{i j}=x_{i}\left(\omega_{j}\right) \in \mathbb{X}_{i}$ with kernel functions $K_{i}\left(x_{i}^{\prime}, x_{i}^{\prime \prime}\right)$ : $\mathbb{X}_{i} \times \mathbb{X}_{i} \rightarrow \mathbb{R}$ defined in them. A broad construction of the SVM was proposed in [5-7] as an instrument of making the Bayesian decision on the discriminant hyperplane $\sum_{i=1}^{n} K_{i}\left(a_{i}, x_{i}\right)+b \gtrless 0$ in the Cartesian product of the kernelinduced hypothetical linear spaces $\boldsymbol{a}=\left(a_{1}, \ldots, a_{n}\right) \in \tilde{\mathbb{X}}_{1} \times \ldots \times \tilde{\mathbb{X}}_{n}, b \in \mathbb{R}$, with an arbitrary a priori density of orientation distribution $\Psi(\boldsymbol{a})=\Psi\left(a_{1}, \ldots, a_{n}\right)$.

It was shown that, under some natural assumptions on the pair of classspecific a priori distribution densities $\varphi(\boldsymbol{x} \mid y= \pm 1,(\boldsymbol{a}, b))$ defined by the same discriminant hyperplane in the combined linear feature space $\boldsymbol{x}=\left(x_{1}, \ldots, x_{n}\right) \in$ $\tilde{\mathbb{X}}_{1} \times \tilde{\mathbb{X}}_{n}$ (see [5-7] for details), the Bayesian estimate of the hyperplane parameters $(\boldsymbol{a}, b)=\left(a_{1}, \ldots, a_{n}, b\right)$ is the solution of the following optimization problem:

$$
\left\{\begin{array}{l}
-\ln \Psi\left(a_{1}, \ldots, a_{n}\right)+c \sum_{j=1}^{N} \delta_{j} \rightarrow \min \left(a_{i} \in \tilde{\mathbb{X}}_{i}, b \in \mathbb{R}, \delta_{j} \in \mathbb{R}\right), \\
y_{j}\left(\sum_{i=1}^{n} a_{i} x_{i j}+b\right) \geqslant 1-\delta_{j}, \delta_{j} \geqslant 0, j=1, \ldots, N
\end{array}\right.
$$

It is only the penalty $-\ln \Psi\left(a_{1}, \ldots, a_{n}\right)$ what distinguishes this generalized training criterion from the classical SVM $\sum_{i=1}^{n} a_{i}^{2}+C \sum_{j=1}^{N} \delta_{j} \rightarrow \min \left(\boldsymbol{a}=\left(a_{1}, \ldots, a_{n}\right) \in\right.$ $\left.\mathbb{R}^{n}, b \in \mathbb{R}, \delta_{1}, \ldots, \delta_{n} \in \mathbb{R}\right)$ for real-valued feature vectors $\boldsymbol{x}_{j}=\left(x_{1 j}, \ldots, x_{n j}\right) \in \mathbb{R}^{n}$.

Two parametric families of a priori densities $\Psi\left(a_{1}, \ldots, a_{n} \mid \mu\right)$ were proposed in [6] as two different means of endowing the training criterion (1) with the ability to emphasize informative object-representation modalities and suppress redundant ones under the desired selectivity level which grows with growing parameter $\mu \geqslant 0$ starting from the full absence of selectivity, namely, retaining all the original modalities when $\mu=0$.

These two parametric families had led in [6] to different modality-selective training criteria named the Relevance Kernel Machine (RKM) with supervised selectivity

$$
\left\{\begin{array}{l}
J_{R K M}\left(a_{1}, r_{1}, \ldots, a_{n}, r_{n}, b, \delta_{1}, \ldots, \delta_{N} \mid \mu\right)= \\
\sum_{i=1}^{n}\left[\left(1 / r_{i}\right)\left(K_{i}\left(a_{i}, a_{i}\right)+1 / \mu\right)+(1 / \mu+1+\mu) \ln r_{i}\right]+ \\
C \sum_{j=1}^{N} \delta_{j} \rightarrow \min \left(a_{i} \in \tilde{\mathbb{X}}_{i}, r_{i} \in \mathbb{R}, b \in \mathbb{R}, \delta_{j} \in \mathbb{R}\right), \\
y_{j}\left(\sum_{i=1}^{n} K_{i}\left(a_{i}, x_{i j}\right)+b\right) \geqslant 1-\delta_{j}, \delta_{j} \geqslant 0, j=1, \ldots, N, r_{i} \geqslant \varepsilon>0, i=1, \ldots, n,
\end{array}\right.
$$

and the Support Kernel Machine (SKM) with supervised selectivity 


$$
\left\{\begin{array}{l}
J_{S K M}\left(a_{1}, \ldots, a_{n}, b, \delta_{1}, \ldots, \delta_{N} \mid \mu\right)= \\
\sum_{i=1}^{n} q\left(a_{i} \mid \mu\right)+C \sum_{j=1}^{N} \delta_{j} \rightarrow \min \left(a_{i} \in \tilde{\mathbb{X}}_{i}, b \in \mathbb{R}, \delta_{j} \in \mathbb{R}\right), \\
q\left(a_{i} \mid \mu\right)= \begin{cases}2 \mu \sqrt{K_{i}\left(a_{i}, a_{i}\right)} & \text { if } \sqrt{K_{i}\left(a_{i}, a_{i}\right)} \leqslant \mu, \\
\mu^{2}+K_{i}\left(a_{i}, a_{i}\right) & \text { if } \sqrt{K_{i}\left(a_{i}, a_{i}\right)}>\mu,\end{cases} \\
y_{j}\left(\sum_{i=1}^{n} K_{i}\left(a_{i}, x_{i j}\right)+b\right) \geqslant 1-\delta_{j}, \delta_{j} \geqslant 0, j=1, \ldots, N .
\end{array}\right.
$$

We consider here only these two training criteria themselves and omit the Bayesian reasoning of their resulting from respective a priori assumptions. The statistical justification is to be found in [6].

The Relevance Kernel Machine (2) and the Support Kernel Machine (3) are generalized versions of the classical SVM which implement two different principles of kernel-based modality selection.

The RKM emphasizes some modalities and relatively suppresses the others by assigning continuous positive weights $r_{i}>0$ to the respective kernels $i \in I=$ $\{1, \ldots, n\}$ in the resulting discriminant hyperplane

$$
\sum_{j: \lambda_{j}>0} y_{j} \lambda_{j} \sum_{i \in I} r_{i} K_{i}\left(x_{i j}, x_{i}\right)+b \gtrless 0
$$

applicable to any new object $\boldsymbol{x}(\omega)=\left(x_{i}(\omega) \in \mathbb{X}_{i}, i=1, \ldots, n\right)$.

On the contrary, the SKM displays a pronounce inclination to complete exclusion of a part of kernels. It partitions the entire set of modality-specific kernels into two subsets, that of support kernels $I_{\text {supp }}=\left\{i: r_{i}>0\right\} \subseteq I$, which occur in the resulting discriminant hyperplane, and that of excluded ones $I \backslash I_{\text {supp }}=\left\{i: r_{i}=0\right\}$.

\section{A smooth dual formulation of the nondifferentiable SKM training problem}

For any training set $\left\{\left(x_{i j}, i \in I\right), y_{j}, j=1, \ldots, N\right\}$, where $I=\{1, \ldots, n\}$ is the set of all modalities, the objective function $J_{S K M}\left(a_{i}, i \in I, b, \delta_{j}, j=1, \ldots N \mid \mu\right)$ in (3) is convex in its range of definition $\tilde{\mathbb{X}}_{1} \times \tilde{\mathbb{X}}_{n} \times \mathbb{R} \times \mathbb{R}^{N}$, and the inequality constraints carve out a convex region in it. Thus, the SKM problem is that of convex optimization.

We denote as $\lambda_{j} \geqslant 0$ and $\pi_{j} \geqslant 0$ the Lagrange multipliers at the inequality constraints, respectively, $y_{j}\left(\sum_{i=1}^{n} K_{i}\left(a_{i}, x_{i j}\right)+b\right)-1+\delta_{j} \geqslant 0$ and $\delta_{j} \geqslant 0$. The convex problem (3) can be shown to be a regular one [10], and, so, it is equivalent to that of finding the saddle point of its Lagrangian

$$
\begin{gathered}
L\left(a_{i}, i \in I, b, \delta_{j}, \lambda_{j}, \pi_{j}, j=1, \ldots N \mid \mu\right)=\frac{1}{2} J_{S K M}\left(a_{1}, \ldots, a_{n}, b, \delta_{1}, \ldots, \delta_{N} \mid \mu\right)- \\
-\sum_{j=1}^{N} \pi_{j} \delta_{j}-\sum_{j=1}^{N} \lambda_{j}\left[y_{j}\left(\sum_{i \in I} K_{i}\left(a_{i}, x_{i j}\right)+b\right)-1+\delta_{j}\right] \rightarrow \\
\rightarrow\left\{\begin{array}{l}
\min \left(a_{i} \in \tilde{\mathbb{X}}_{i}, i \in I, b \in \mathbb{R}, \delta_{j} \in \mathbb{R}, j=1, \ldots, N\right), \\
\max \left(\pi_{j} \geqslant 0, \lambda_{j} \geqslant 0, j=1, \ldots, N\right) .
\end{array}\right.
\end{gathered}
$$


If $\left[\left(\tilde{a}_{i}, i \in I, \tilde{b}, \tilde{\delta}_{j},, j=1, \ldots N\right) ;\left(\tilde{\lambda}_{j}, \tilde{\pi}_{j}, j=1, \ldots N\right)\right]$ is a saddle point, its left part $\left(\tilde{a}_{i}, i \in I, \tilde{b}, \tilde{\delta}_{j},, j=1, \ldots N\right)$ is a solution of the SKM problem (3), and vice versa, each of its solutions $\left(\tilde{a}_{i}, i \in I, \tilde{b}, \tilde{\delta}_{j},, j=1, \ldots N\right)$ is the left part of a saddle point of the Lagrangian (5).

Expanding the objective function in (5) in accordance with (3) gives the detailed expression of the Lagrangian:

$L\left(a_{i}, i \in I, b, \delta_{j}, \lambda_{j}, \pi_{j}, j=1, \ldots N \mid \mu\right)=$

$\frac{1}{2}\left(\sum_{i \in I} q\left(a_{i} \mid \mu\right)+C \sum_{j=1}^{N} \delta_{j}\right)-\sum_{j=1}^{N} \pi_{j} \delta_{j}-\sum_{j=1}^{N} \lambda_{j}\left[y_{j}\left(\sum_{i \in I} K_{i}\left(a_{i}, x_{i j}\right)+b\right)-1+\delta_{j}\right]$.

It is convenient to introduce special notations for each sum of constituents that depend on the $i$ th modality-specific element $a_{i}$ of the entire direction vector $\boldsymbol{a}=\left(a_{1}, \ldots, a_{n}\right)$ :

$$
L_{i}\left(a_{i}, \lambda_{j}, j=1, \ldots, N \mid \mu\right)=\frac{1}{2} q\left(a_{i} \mid \mu\right)-K_{i}\left(a_{i}, \sum_{j=1}^{N} y_{j} \lambda_{j} x_{i j}\right) .
$$

In these terms, the Lagrangian (5) or (6) will have the form

$$
\begin{aligned}
& L\left(a_{i}, i \in I, b, \delta_{j}, \lambda_{j}, \pi_{j}, j=1, \ldots N \mid \mu\right)= \\
& \left.\sum_{i \in I} L_{i}\left(a_{i}, \lambda_{j}, j=1, \ldots, N \mid \mu\right)+\sum_{j=1}^{N}\left(\frac{C}{2}-\pi_{j}-\lambda_{j}\right)\right) \delta_{j}- \\
& -\left(\sum_{j=1}^{N} y_{j} \lambda_{j}\right) b+\sum_{j=1}^{N} \lambda_{j} .
\end{aligned}
$$

Finding the saddle point of the Larangian is equivalent to maximizing the dual function of the Lagrange multipliers

$$
\begin{aligned}
& W\left(\lambda_{j}, \pi_{j}, j=1, \ldots, N \mid \mu\right)= \\
& \sum_{j=1}^{N} \lambda_{j}+\min _{a_{i} \in \tilde{\mathbb{X}}_{i}, b \in \mathbb{R}, \delta_{j} \in \mathbb{R}} L\left(a_{i}, i \in I, b, \delta_{j}, \lambda_{j}, \pi_{j}, j=1, \ldots N \mid \mu\right) .
\end{aligned}
$$

However, the minimum value of the second term in (8) exists only if the Lagrange multipliers satisfy the inequalities $C / 2-\pi_{j}-\lambda_{j}=0$, or, on the force of the restrictions $\pi_{j} \geqslant 0$,

$$
0 \leqslant \lambda_{j} \leqslant \frac{C}{2}, j=1, \ldots, N .
$$

Analogously, the third term of (8) has the minimum only if

$$
\sum_{j=1}^{N} y_{j} \lambda_{j}=0 .
$$

Thus, the dual function

$$
W\left(\lambda_{j}, j=1, \ldots, N \mid \mu\right)=\sum_{j=1}^{N} \lambda_{j}+\sum_{i \in I} \min _{a_{i} \in \tilde{\mathbb{X}}_{i}} L_{i}\left(a_{i}, \lambda_{j}, j=1, \ldots, N \mid \mu\right)
$$


is to be maximized under constraints (10) and (11).

To accomplish formulation of the dual problem, it is required to find how the minimum values of the functions $L_{i}\left(a_{i}, \lambda_{j}, j=1, \ldots, N \mid \mu\right)(7)$ with respect to $a_{i}$ depend on the Lagrange multipliers $\lambda_{i}$ for each of the modalities $i \in I$. But these functions contain, in their turn, nondifferentiable functions $q\left(a_{i} \mid \mu\right)$ (3), what leads to the necessity of using the notions of subgradient and subdifferential, instead of the usual gradient, to formulate the minimum condition of a convex function [10].

Definition 1. Vector $d \in \tilde{\mathbb{X}}$ in a linear space $\tilde{\mathbb{X}}$ with inner product $K\left(x^{\prime}, x^{\prime \prime}\right)$ is called a subgradient of convex function $f: \tilde{\mathbb{X}} \rightarrow \mathbb{R}$ at point $a \in \tilde{\mathbb{X}}$ if the inequality $f(x)-f(a) \geqslant K(d, x-a)$ holds for all $x \in \tilde{\mathbb{X}}$.

Definition 2. The set of all subgradients of convex function $f: \tilde{\mathbb{X}} \rightarrow \mathbb{R}$ at point $a \in \tilde{\mathbb{X}}$ is called the subdifferential $\partial f(a) \subseteq \tilde{\mathbb{X}}$ at this point.

Property. The condition that the subdifferential at point $a \in \tilde{\mathbb{X}}$ contains the null element $\phi \in \partial f(a) \subseteq \tilde{\mathbb{X}}$ is necessary and sufficient for this point to be a minimum point of convex function $f$.

The latter property creates a mathematical basis for a closed form of the smooth optimization problem (12) dual to the original nondifferentiable SKM problem (3). This is a problem of maximizing a linear function of $N+n$ variables, namely, $N$ Lagrange multipliers $\lambda_{j}$ and $n$ auxiliary variables $\xi_{i}$, under quadratic and linear constraints.

Theorem 1. The problem

$$
\left\{\begin{array}{l}
W\left(\xi_{i}, i \in I, \lambda_{j}, j=1, \ldots, N\right)=\frac{1}{2} \sum_{i \in I} \xi_{i}+C \sum_{j=1}^{N} \lambda_{j} \rightarrow \max , \\
\xi_{i} \leqslant \mu^{2}-\sum_{j=1}^{N} \sum_{l=1}^{N} y_{j} y_{l} K_{i}\left(x_{i j}, x_{i l}\right) \lambda_{j} \lambda_{l}, \quad \xi_{i} \leqslant 0, \quad i \in I, \\
\sum_{j=1}^{N} y_{j} \lambda_{j}=0 ; 0 \leqslant \lambda_{j} \leqslant \frac{C}{2}, \quad j=1, \ldots, N,
\end{array}\right.
$$

is dual to the SKM training problem (3).

The proof leans upon the following lemma which is a result of immediate application of the above formulated property of an arbitrary nondifferentiable convex function to the functions $L_{i}\left(a_{i}, \lambda_{j}, j=1, \ldots, N \mid \mu\right)$ in (12).

Lemma 1. The minimum of function $L_{i}\left(a_{i}, \lambda_{j}, j=1, \ldots, N \mid \mu\right)$ (7) with respect to variable $a_{i} \in \tilde{\mathbb{X}}_{i}$ is reached at the points

$$
\begin{cases}\tilde{a}_{i}=\eta_{i} \sum_{j=1}^{N} y_{j} \lambda_{j} x_{i j}, \quad r_{i}=1, & \text { if } K_{i}\left(\sum_{j=1}^{N} y_{j} \lambda_{j} x_{i j}, \sum_{j=1}^{N} y_{j} \lambda_{j} x_{i j}\right)>\mu^{2}, \\ \tilde{a}_{i}=\eta_{i} \sum_{j=1}^{N} y_{j} \lambda_{j} x_{i j}, 0 \leqslant r_{i} \leqslant 1, & \text { if } K_{i}\left(\sum_{j=1}^{N} y_{j} \lambda_{j} x_{i j}, \sum_{j=1}^{N} y_{j} \lambda_{j} x_{i j}\right)=\mu^{2}, \\ \tilde{a}_{i}=\phi_{i}, & \text { if } K_{i}\left(\sum_{j=1}^{N} y_{j} \lambda_{j} x_{i j}, \sum_{j=1}^{N} y_{j} \lambda_{j} x_{i j}\right)<\mu^{2},\end{cases}
$$


in the sense of the linear operations and the null element induced by the respective kernel $K_{i}\left(x^{\prime}, x^{\prime \prime}\right)$ in the hypothetical linear space $\tilde{\mathbb{X}}_{i}$. At each of such points,

$$
\begin{aligned}
& \min _{a_{i} \in \tilde{\mathbb{X}}_{i}} L_{i}\left(a_{i}, \lambda_{j}, j=1, \ldots, N \mid \mu\right)=L_{i}\left(\tilde{a}_{i}, \lambda_{j}, j=1, \ldots, N \mid \mu\right)= \\
& \frac{1}{2} \min \left\{0 ; \mu^{2}-K_{i}\left(\sum_{j=1}^{N} y_{j} \lambda_{j} x_{i j}, \sum_{j=1}^{N} y_{j} \lambda_{j} x_{i j}\right)\right\} .
\end{aligned}
$$

\section{The resulting discriminant hyperplane and support kernels}

Let the dual optimization problem (13) have been solved. Only the Lagrange multipliers $\lambda_{1} \geqslant 0, \ldots, \lambda_{N} \geqslant 0$ are of interest, the auxiliary values $\pi_{1} \leqslant 0, \ldots, \pi_{n} \leqslant 0$ may be dropped. In accordance with (14), the found solution partitions the set of all kernels $I=\{1, \ldots, n\}$ into three subsets:

$$
\begin{aligned}
& I^{+}=\left\{i \in I: \sum_{j=1}^{N} \sum_{l=1}^{N} y_{j} y_{l} K_{i}\left(x_{i j}, x_{i l}\right) \lambda_{j} \lambda_{l}>\mu^{2}\right\}, \\
& I^{0}=\left\{i \in I: \sum_{j=1}^{N} \sum_{l=1}^{N} y_{j} y_{l} K_{i}\left(x_{i j}, x_{i l}\right) \lambda_{j} \lambda_{l}=\mu^{2}\right\}, \\
& I^{-}=\left\{i \in I: \sum_{j=1}^{N} \sum_{l=1}^{N} y_{j} y_{l} K_{i}\left(x_{i j}, x_{i l}\right) \lambda_{j} \lambda_{l}<\mu^{2}\right\} .
\end{aligned}
$$

Theorem 2. The optimal discriminant hyperplane defined by the solution of the SKM training problem (3) has the form

$$
\sum_{j: \lambda_{j}>0} y_{j} \lambda_{j}\left(\sum_{i \in I^{+}} K_{i}\left(x_{i j}, x_{i}\right)+\sum_{i \in I^{0}} r_{i} K_{i}\left(x_{i j}, x_{i}\right)\right)+b \gtrless 0,
$$

where the numerical parameters $\left\{0 \leqslant r_{i} \leqslant 1, i \in I^{0} ; b\right\}$ are solutions of the linear programming problem

$\left\{\begin{array}{l}2 \mu^{2} \sum_{i \in I^{0}} r_{i}+C \sum_{j=1}^{n} \delta_{j} \rightarrow \min \left(r_{i}, i \in I^{0} ; b ; \delta_{1}, \ldots, \delta_{N}\right), \\ \sum_{i \in I^{0}}\left(\sum_{l=1}^{N} y_{j} y_{l} K_{i}\left(x_{i j}, x_{i l}\right) \lambda_{l}\right) r_{i}+y_{j} b+\delta_{j} \geqslant 1-\sum_{i \in I^{+}} \sum_{l=1}^{N} y_{j} y_{l} K_{i}\left(x_{i j}, x_{i l}\right) \lambda_{l}, \\ \delta_{j} \geqslant 0, j=1, \ldots, N, \quad 0 \leqslant r_{i} \leqslant 1, i \in I^{0} .\end{array}\right.$

\section{The subset of support kernels}

The solution $\left(\hat{r}_{i}, i \in I^{0} ; \hat{b} ; \hat{\delta}_{1}, \ldots, \hat{\delta}_{N}\right)$ of the linear programming problem (18) is conmpletely defined by the training set $\mathcal{X}=\left\{\boldsymbol{x}_{j}=\left(x_{1 j}, \ldots, x_{n j}\right), y_{j}, j=\right.$ $1, \ldots, N\} \in \mathbb{X}_{1} \times \ldots \times \mathbb{X}_{n} \subseteq \tilde{\mathbb{X}}_{1} \times \ldots \times \tilde{\mathbb{X}}_{n}$. As it is seen from the criterion (18), some of coefficients $\left(\hat{r}_{i}, i \in I^{0}\right)$ may equal zero if the respective constraints $0 \leqslant r_{i}$ are active at the solution point. 
However, it can be shown that, if all the linear spaces $\tilde{\mathbb{X}}_{i}$ are finite-dimensional, the subset of such configurations $\{\mathcal{X}\}$ is of zero Lebesgue measure in the linear space $\tilde{\mathbb{X}}_{1} \times \ldots \times \tilde{\mathbb{X}}_{n}$. Thus, if the training set is considered as a random point defined by a continuous probability distribution, the inequalities $\hat{r}_{i}>0$ are met almost surely for all $i \in I^{0}$.

This means that without any loss of generality the constraints $\left\{0 \leqslant r_{i} \leqslant\right.$ $\left.1, i \in I^{0}\right\}$ may be omitted in (18), and, nevertheless, all kernels $i \in I^{0}$ will occur in the discriminant hyperplane (17) with nonzero weights. It is natural to call the subset $I_{\text {supp }}=I^{+} \cup I^{0} \subseteq I$ the set of support kernels .

The structure of the subsets of kernels (16) explicitly reveals how the subset of support kernels $I_{\text {supp }}$ is affected by the parameter $\mu$ in the training criterion (3).

If $\mu=0$, the set of evident support kernels $I^{+} \subseteq I$ coincides with the entire set $I=\{1, \ldots, n\}$. In this particular case, function $q\left(a_{i} \mid \mu\right)$ in (3) is quadratic $q\left(a_{i} \mid \mu\right)=$ const $+K_{i}\left(a_{i}, a_{i}\right)$ for all $a_{i} \in \tilde{\mathbb{X}}_{i}$, the training criterion does not differ from the usual SVM having no selectivity properties, and all the initial kernels are support ones because all of them occur in the resulting decision rule.

As $\mu$ grows, more and more kernels get in the set $I^{-}$of evident nonsupport kernels (16), and, respectively, the set of support ones $I_{\text {supp }}=I^{+} \cup I^{0}$ gets smaller.

A nonlimited growth of the selectivity parameter $\mu \rightarrow \infty$ drives, finally, all the kernels into $I^{-}$, so that no support kernels remain at all $I_{\text {supp }}=\varnothing$.

\section{Adjusting the selectivity parameter}

The selectivity parameter $0 \leqslant \mu<\infty$ is a structural parameter of the SKM training criterion. It determines a sequence of nested classes of training-set models whose dimensionality diminishes as $\mu$ grows, starting from the usual SVM model if $\mu=0$. It is impossible in principle to "estimate" its most appropriate value immediately from the result of training.

At present, the most effective method for choosing the value of a structural parameter is Cross-Validation that is based on directly estimating the generalization performance of the training method.

\section{Experiments on real-world data}

For the real data experiment, we used the lung cancer data set from the UCI repository [11]. The data set contains feature vectors of $N=32$ patients partitioned into two subsets $N_{+1}=9$ and $N_{-1}=23$ with, respectively, diagnosticated and not diagnosticated pathological lung cancer. Each vector consists of $n=56$ features whose number essentially exceeds the size of the available training set.

We have no information on either the individual variables or the origin of the data.

As the data set does not contain a test set, the relationship between the generalization performance of the algorithms and the selectivity level $\mu$ was estimated by the cross-validation method. The results of the experiment are shown in Fig. 1. 


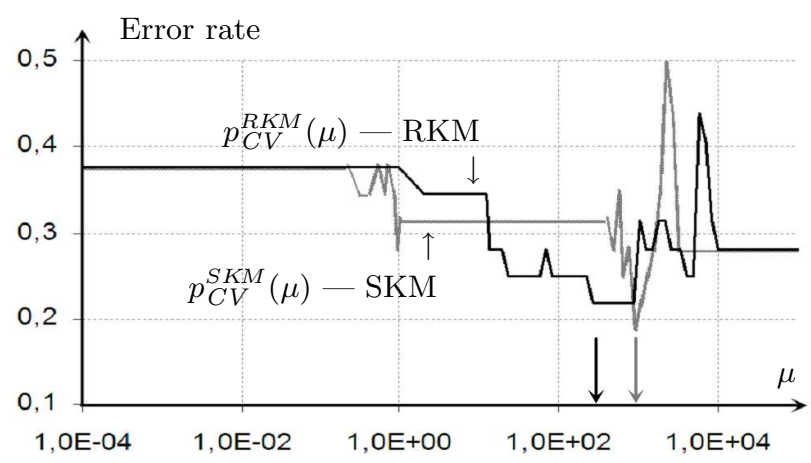

Fig. 1. The result of cross-validation on the lung cancer data set for increasing values of selectivity level $\mu$.

For small values of $\mu$, both techniques are equivalent to the usual SVM applied to all $n=56$ variables, so that, the respective error rates have the same value 0.38 .

The minimum achievable error rate for the RKM is 0.187 , whereas for the SKM it equals 0.219 . For the optimal levels of selectivity $\mu$, both techniques decrease their error rates approximately twice, but the weights estimated by RKM appreciably differ from zero at 4 features from 56, whereas SKM retains only 2 of them. This fact appears to be the cause of some advantage of RKM over SKM with the minimum error rate 0.187 against 0.219 .

Finally, when $\mu$ becomes too large, both RKM and SKM remove all features, and the error rate of recognition tends to the a priori level 0.281 determined by the ratio $N_{1} / N_{-1}$ between the numbers of representatives of the classes in the training set.

\section{Conclusions}

The Support Kernel Machine (SKM) and the Relevance Kernel Machine (RKM) are two different methods of selective combining kernel-based modalities of arbitrary kind in multimodal pattern recognition. The former of them consists in rigidly selecting a subset of presumably informative support kernels and excluding the others, whereas the latter one assigns positive weights to all the kernels.

The names Support Kernel Machine and Relevance Kernel Machine arose from the analogy with the distinction between the Support Vector Machine (SVM) [8] and the Relevance Vector Machine (RVM) [9], which differ from each other by the binary against weighted understanding of occurring the training-set objects in the linear decision rule.

However, it is just the rigid SVM scheme of constructing the training problem which underlies both SKM and RKM methods of selective combining the kernelbased modalities of object representation. Thus, more accurate names, in this case, would be Support Kernel SVM and Relevance Kernel SVM. 
The experiments have shown that the SKM and RKM methods display quite similar generalization performance with a slight quantitative superiority of RKM. But from another point of view, SKM allows to essentially decrease the number of measurements to be done on new objects after completing the training process.

\section{Acknowledgements}

The research leading to these results has received funding from the Russian Foundation for Basic Research, grant no. 08-01-00695-a, and the European Community's Seventh Framework Programme (FP7/2007-2013) under grant agreement no. 215078. We also gratefully acknowledge the support of EPSRC through grant $\mathrm{EP} / \mathrm{F} 069626 / 1$.

\section{References}

1. A. Ross, A. K. Jain. Multimodal biometrics: An overview. Proceedings of the 12th European Signal Processing Conference (EUSIPCO), 2004, Vienna, Austria, pp. 1221-1224.

2. I.M. Guyon, S. R. Gunn, M. Nikravesh, L. Zadeh, Eds. Feature Extraction, Foundations and Applications. Springer, 2006.

3. S. Sonnenburg, G. Rätsch, C. Schäfer. A general and efficient multiple kernel learning algorithm. Proceedings of the 19th Annual Conference on Neural Information Processing Systems, Vancouver, Canada, December 5-8, 2005.

4. V. Sulimova, V. Mottl, A. Tatarchuk. Multi-kernel approach to on-line signature verification. Proceedings of the 8th IASTED International Conference on Signal and Image Processing, Honolulu, Hawaii, USA, August 14-16, 2006.

5. V. Mottl, A. Tatarchuk, V. Sulimova, O. Krasotkina, O. Seredin. Combining pattern recognition modalities at the sensor level via kernel fusion. Proceedings of the 7th International Workshop on Multiple Classifier Systems. Czech Academy of Sciences, Prague, Czech Republic, May 23-25, 2007.

6. A. Tatarchuk, V. Mottl, A. Eliseyev, D. Windridge. Selectivity supervision in combining pattern-recognition modalities by feature- and kernel-selective Support Vector Machines. Proceedings of the 19th International Conference on Pattern Recognition, Tampa, USA, December 8-11, 2008.

7. A. Tatarchuk, V. Sulimova, D. Windridge, V. Mottl, M. Lange. Supervised selective combining pattern recognition modalities and its application to signature verification by fusing on-line and off-line kernels. Proceedings of the 8th International Workshop on Multiple Classifier Systems. University of Iceland, Reykjavik, Iceland, June 10-12, 2009.

8. V. Vapnik. Statistical Learning Theory. John-Wiley \& Sons, Inc. 1998.

9. C.M. Bishop, M.E. Tipping. Variational relevance vector machines. Proceedings of the 16th Conference on Uncertainty in Artificial In-telligence. Morgan Kaufmann, 2000, pp. 46-53.

10. J.-B. Hiriart-Urruty, C. Lemarechal. Fundamentals of Convex Analysis. Springer, 2001.

11. UCI Machine Learning Repository: Lung Cancer Data Set. http://archive.ics.uci.edu/ml/datasets/Lung+Cancer 